Trinity University

Digital Commons @ Trinity

Physics and Astronomy Faculty Research

Physics and Astronomy Department

$5-2014$

Born-Oppenheimer Study of Two-Component Few-Particle Systems Under One-Dimensional Confinement

Nirav P. Mehta

Trinity University, nmehta@trinity.edu

Follow this and additional works at: https://digitalcommons.trinity.edu/physics_faculty

Part of the Physics Commons

Repository Citation

Mehta, N.P. (2014). Born-Oppenheimer study of two-component few-particle systems under onedimensional confinement. Physical Review A, 89(5), 052706. doi: 10.1103/PhysRevA.89.052706

This Article is brought to you for free and open access by the Physics and Astronomy Department at Digital Commons @ Trinity. It has been accepted for inclusion in Physics and Astronomy Faculty Research by an authorized administrator of Digital Commons @ Trinity. For more information, please contact jcostanz@trinity.edu. 


\title{
Born-Oppenheimer study of two-component few-particle systems under one-dimensional confinement
}

\author{
N. P. Mehta* \\ Department of Physics and Astronomy, Trinity University, San Antonio, Texas 78212-7200, USA \\ (Received 14 January 2014; revised manuscript received 20 April 2014; published 13 May 2014)
}

\begin{abstract}
The energy spectrum, atom-dimer scattering length, and atom-trimer scattering length for systems of three and four ultracold atoms with $\delta$-function interactions in one dimension are presented as a function of the relative mass ratio of the interacting atoms. The Born-Oppenheimer approach is used to treat three-body ("HHL") systems of one light and two heavy atoms, as well as four-body ("HHHL") systems of one light and three heavy atoms. Zero-range interactions of arbitrary strength are assumed between different atoms, but the heavy atoms are assumed to be noninteracting among themselves. Fermionic and bosonic heavy atoms with both positive and negative parity are considered.
\end{abstract}

DOI: 10.1103/PhysRevA.89.052706

PACS number(s): $34.50 . \mathrm{Cx}, 37.10 . \mathrm{Jk}$

\section{INTRODUCTION}

Cold-atom experiments now have the ability to simultaneously control the atom-atom scattering length and the trapping geometry. Quantum gases with essentially zero-range interactions in one-dimensional (1D) trap geometries have been realized [1-6]. At the same time, the variety of atomic species that have been trapped and cooled, including all of the alkali metals, continues to grow, ranging in mass from hydrogen [7] to radium [8]. Moreover, quantum degenerate mixtures of atoms have been the subject of several experiments related to, for example, the creation of a gas of degenerate polar molecules [9], the observation of heteronuclear Efimov states [10], and the realization of mixtures of alkali atoms with alkaline-earth-like atoms [11].

These recent experimental advances were preceded by a large body of literature on the few- and many-body physics of strongly interacting 1D systems [12-17]. More recent theory work includes the calculation of the three-boson hyperradial potential curves [18-20], three-body recombination rates and threshold laws [21], and benchmark quality hyperspherical calculations of three-boson binding energies and scattering amplitudes [22]. The three-body problem for unequal masses has been studied in free space [23] and in an optical lattice [24].

Of particular relevance to the present study is the massdependent calculation of atom-dimer $(2+1)$ scattering lengths and three-body binding energies performed in [23]. The calculations of [23] incorporate all of the adiabatic hyperspherical potential curves necessary for numerical convergence. Here, instead of the (in principle) exact adiabatic hyperspherical representation [25], we use the Born-Oppenheimer approach. For the three-body calculations presented here, the accuracy of the Born-Oppenheimer factorization is studied by direct comparison to the results of [23], and that comparison gives some quantitative insight to the accuracy of the four-body calculations that follow.

It should also be noted that the HHHL system for spinpolarized heavy fermions in three dimensions (3D) has been studied by Castin et al. [26]. They found that for heavy fermions with $J^{\Pi}=1^{+}$symmetry, an infinite set of four-body

*nmehta@trinity.edu states appears in the mass range $13.384<m_{H} / m_{L}<13.607$. Castin et al. argue that these states have Efimov character; however, there seems to be some debate in the literature. Other authors [27] have argued these are truly new states with properties distinct from Efimov states. The authors of [27] consider particles interacting with attractive $1 / r^{2}$ interactions, basing their model on a Born-Oppenheimer calculation of the potential energy surface governing the heavy-particle dynamics. Better establishing the accuracy of the Born-Oppenheimer approximation for short-range potentials could potentially play a role in the interpretation of these calculations.

The Born-Oppenheimer approach has been successfully applied to cold-atom systems in optical lattices to study crystalline phases in Fermi mixtures [28]. The authors of [28] note that the large mass ratios needed to observe these crystalline phases can be achieved with small filling factors by tuning the effective mass for the heavy particles. We note that such a scheme could potentially be used to observe the tetramer states predicted in this work.

In this paper, we consider 1D systems of three and four particles in which one particle is "light" (of mass $m_{L}=\beta m_{H}$ with $0<\beta<1$ ) in comparison to the remaining "heavy" (mass $m_{H}$ ) particles. We restrict our attention to cases of noninteracting heavy particles $\left(a_{H H} \rightarrow \infty\right)$. Here, $a_{H H}$ is the 1D heavy-heavy scattering length. We denote the 1D heavylight scattering length simply by $a$. For cylindrical harmonic traps in which only the lowest transverse mode is significantly populated, the $1 \mathrm{D}$ scattering length may be expressed in terms of the 3D $s$-wave scattering length $a_{3 \mathrm{D}}$ and the transverse oscillator length $a_{\perp}$ by the Olshanii formula [29,30]:

$$
a=-\frac{a_{\perp}^{2}}{2 a_{3 \mathrm{D}}}\left(1-C \frac{a_{3 \mathrm{D}}}{a_{\perp}}\right),
$$

where $C \approx 1.4603$. Equation (1) incorporates the effect of virtual transitions to excited transverse modes. When $a_{\perp}=$ $C a_{3 \mathrm{D}}$, Eq. (1) predicts a "confinement induced resonance" (CIR), and the 1D scattering length vanishes.

The degree to which the renormalization of the 1D atomatom scattering length by Eq. (1) accounts for the quasi-1D nature of the confinement in few-body calculations is not a trivial question [31-33]. Fully quasi-1D few-body calculations are complicated by the fact that cylindrical confinement breaks 
spherical symmetry, and the total angular momentum of the three- or four-body system is not a good quantum number. In this paper, we proceed under the assumption that meaningful few-body observables may be calculated with purely 1D $\delta$ function interactions, renormalized according to Eq. (1).

This paper is organized as follows. In Sec. II, we calculate the Born-Oppenheimer potential curve describing the effective heavy-heavy interaction as mediated by the light particle. The HHL bound-state spectrum and the H-HL scattering length is calculated as a function of the heavy-light mass ratio. The accuracy of the Born-Oppenheimer approximation is studied by comparison to the high-accuracy calculation of [23].

In Sec. III, we calculate the two-dimensional potentialenergy surface describing the heavy-particle dynamics in the HHHL system. The adiabatic wave function describing the light particle is governed by a one-dimensional Schrödinger equation with three $\delta$ functions. We choose coordinates such that for a given permutation of heavy particles, the ordering of the $\delta$ functions along the light-particle coordinate is fixed. The resulting energy surface is then used in a calculation of the three-body adiabatic hyperradial potential curves for the heavy particles. From those potential curves, the HHHL binding energies and H-HHL scattering lengths are calculated.

\section{THREE-BODY (HHL) PROBLEM}

Let particles 1 and 2 have mass $m_{1}=m_{2}=m_{H}$ and particle 3 have mass $m_{L}=\beta m_{H}$. Throughout this paper, we set $\hbar=1$. For a zero-range heavy-light interaction of the form $V_{i j}=g \delta\left(x_{i}-x_{j}\right)$, the 1D H-L scattering length is $a=-1 /\left(\mu_{\mathrm{HL}} g\right)$, and assuming $a>0$, the heavy-light binding energy is $B_{2}=\mu_{\mathrm{HL}} g^{2} / 2=1 /\left(2 \mu_{\mathrm{HL}} a^{2}\right)$. For particle positions $\left\{x_{1}, x_{2}, x_{3}\right\}$, we introduce the following unitless mass-scaled Jacobi coordinates (see Fig. 3):

$$
\begin{aligned}
& x=\frac{1}{a} \sqrt{\frac{\mu_{12}}{\mu_{3 b}}}\left(x_{2}-x_{1}\right), \\
& y=\frac{1}{a} \sqrt{\frac{\mu_{12,3}}{\mu_{3 b}}}\left(\frac{m_{1} x_{1}+m_{2} x_{2}}{m_{1}+m_{2}}-x_{3}\right) .
\end{aligned}
$$

Here, $\mu_{12}=m_{H} / 2, \quad \mu_{12,3}=m_{H}[2 \beta /(2+\beta)]$, and $\mu_{3 b}=$ $\sqrt{\mu_{12} \mu_{12,3}}$ are reduced masses. The heavy-light reduced mass is $\mu_{\mathrm{HL}}=m_{H}[\beta /(1+\beta)]$. It is convenient to scale the Hamiltonian by the heavy-light binding energy:

$$
B_{2}=\frac{1}{m_{H} a^{2}} \frac{\beta+1}{2 \beta},
$$

so that all energies are measured in units of $B_{2}$. The Schrödinger equation then reads

$$
\begin{aligned}
& -\frac{1}{2 \mu_{3}}\left(\frac{\partial^{2}}{\partial x^{2}}+\frac{\partial^{2}}{\partial y^{2}}\right) \Psi(x, y)+g_{3}\left[\lambda \delta\left(2 x_{0}\right)+\delta\left(y+x_{0}\right)\right. \\
& \left.\quad+\delta\left(y-x_{0}\right)\right] \Psi(x, y)=E \Psi(x, y) .
\end{aligned}
$$

The parameter $\lambda$ is the ratio of the heavy-heavy coupling to the heavy-light coupling. In this work, only $\lambda \rightarrow 0$ and $\lambda \rightarrow \infty$ are considered. The notational cost of scaling by $B_{2}$ is contained in the definition of the following unitless parameters:

$$
\begin{aligned}
& \mu_{3}=\frac{1+\beta}{2 \sqrt{\beta(2+\beta)}}, \\
& g_{3}=-2 \sqrt{2}\left(\frac{\beta}{2+\beta}\right)^{1 / 4}, \\
& x_{0}=x \sqrt{\frac{\beta}{2+\beta}} .
\end{aligned}
$$

We now assume the wave function may be approximated by the Born-Oppenheimer product:

$$
\Psi(x, y)=\Phi(x ; y) \psi(x),
$$

where $\Phi(x ; y)$ is a solution to the fixed- $x$ equation,

$$
\begin{aligned}
& {\left[\frac{-1}{2 \mu_{3}} \frac{\partial^{2}}{\partial y^{2}}+g_{3}\left[\delta\left(y+x_{0}\right)+\delta\left(y-x_{0}\right)\right]\right] \Phi(x ; y)} \\
& \quad=u(x) \Phi(x ; y)
\end{aligned}
$$

and $u(x)<0$ is the Born-Oppenheimer potential in units of the H-L binding energy. Note that the solutions $\Phi(x ; y)$ and the potential curve $u(x)$ are independent of $\lambda$. Inserting Eq. (9) into Eq. (5) and making use of Eq. (10) yields

$$
\left(\frac{-1}{2 \mu_{3}} \frac{\partial^{2}}{\partial x^{2}}+g_{3} \lambda \delta\left(2 x_{0}\right)+u(x)+\frac{\tilde{Q}(x)}{2 \mu_{3}}\right) \psi(x)=E \psi(x),
$$

where

$$
\tilde{Q}(x)=\left\langle\frac{\partial \Phi}{\partial x} \mid \frac{\partial \Phi}{\partial x}\right\rangle_{y} .
$$

It is understood that the integration in the matrix element $\tilde{Q}(x)$ is carried out over the $y$ coordinate only, while the adiabatic coordinate $x$ is held fixed.

\section{A. Solution to the adiabatic equation}

Equation (10) is symmetric with respect to the operation $y \rightarrow-y$, and so the eigenstates $\Phi(x ; y)$ must be even or odd under that operation. The elementary solutions that vanish as $|y| \rightarrow \infty$ are conveniently written for positive $y$ as

$$
\Phi(x ; y)= \begin{cases}A \sinh (\kappa y)+B \cosh (\kappa y), & \text { if } 0 \leqslant y \leqslant x_{0}, \\ D e^{-\kappa y}, & \text { if } x_{0} \leqslant y,\end{cases}
$$

where $\kappa(x)=\sqrt{-2 \mu_{3} u(x)}$. For the even solution, $A=0$, while for the odd solution, $B=0$. Matching the wave functions, and imposing the derivative discontinuity across the $\delta$ function at $y=x_{0}$ leads to the following transcendental equation for the eigenvalue $\kappa$ :

$$
\frac{\kappa}{g_{3} \mu_{3}}+1=(-1)^{P+1} e^{-2 \kappa x_{0}} .
$$

Here, $P=0$ corresponds to the (even) solution for which $\left.\frac{\partial \Phi}{\partial y}\right|_{y=0}=0$, and $P=1$ corresponds to the (odd) solution for which $\left.\Phi\right|_{y=0}=0$. Borrowing language from molecular physics, one can view the $P=0$ solution as belonging to the "bonding" orbital, and the $P=1$ solution to the "antibonding" orbital. 

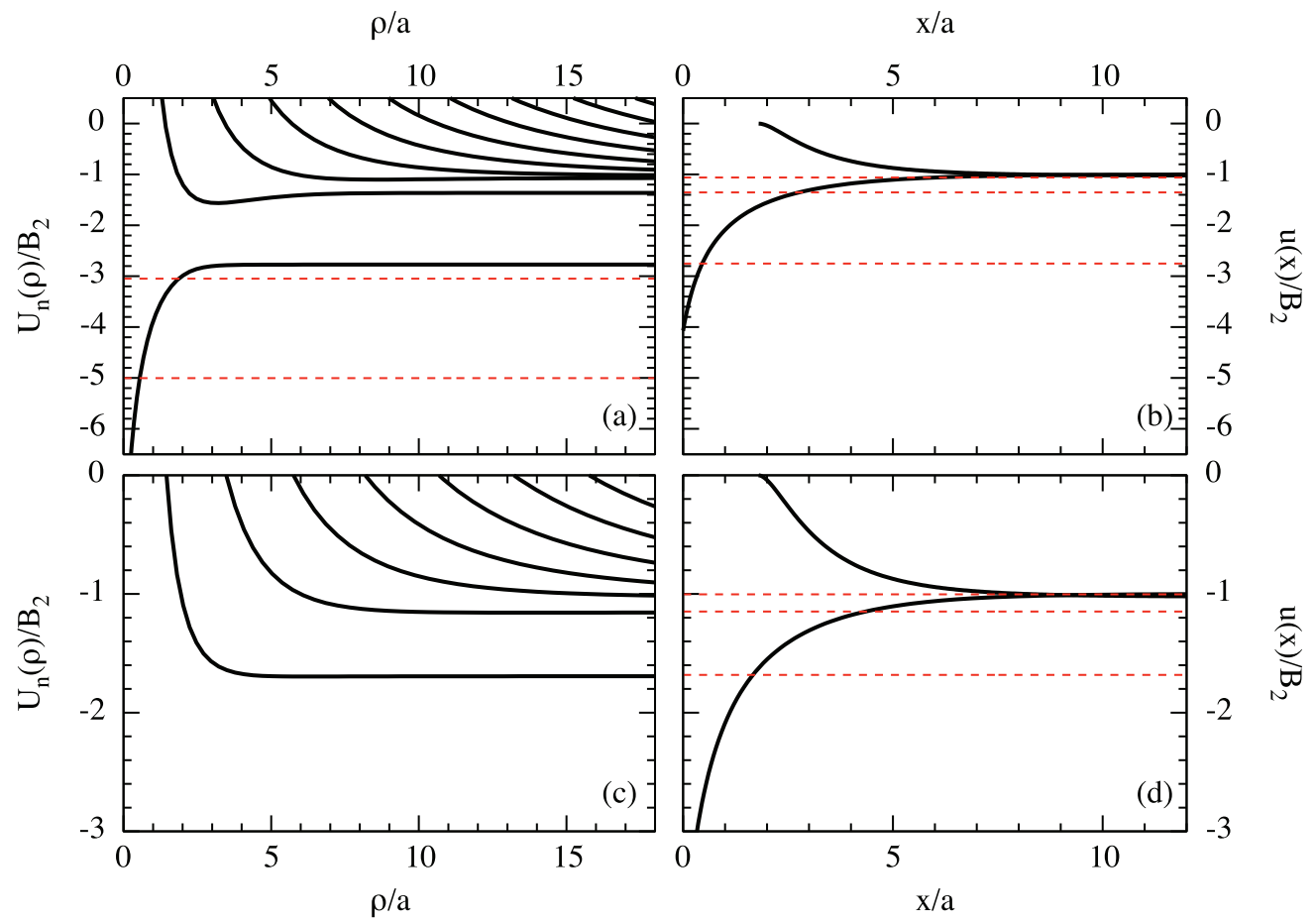

FIG. 1. (Color online) Graph (a) shows the bosonic hyperradial potential curves describing the heavy-particle dynamics in the HHHL system, while graph (b) shows the corresponding Born-Oppenheimer potential curves for the HHL system. Graphs (c) and (d) show similar curves, except for fermions. All graphs are for $\beta^{-1}=22.08$, appropriate for Li-Cs mixtures. The dashed-red lines indicate bound states.

The potential curves resulting from the $x$-dependent solution to Eq. (14) for $\beta^{-1}=22.08$ (for Li-Cs mixtures) are shown in Figs. 1(b) and 1(d). The potential curves shown in these two graphs are identical because Eq. (5) is independent of the heavy-particle symmetry. Any apparent differences are due to the energy scales on the graph. The bound-state structure, however, is dependent on the heavy symmetry through the boundary condition placed on $\psi(x)$ at $x=0$. Note that the $\lambda=\infty$ solutions to Eq. (11) for heavy bosons are identical to those for noninteracting heavy fermions. The boundary condition $\psi(0)=0$ is applied for fermionic heavy atoms as well as fermionized bosonic atoms, leading to the correspondence first recognized in Ref. [34]. For small heavy-atom separations, there is no $P=1$ negative-energy solution to Eq. (14), and the light particle is lost to the continuum where the excited-state potential terminates at the zero-energy threshold.

The atom-dimer scattering length and the HHL spectrum are to a very good approximation determined solely by the potential curve corresponding to the $P=0$ solution to Eq. (14), so we shall restrict our immediate focus to that solution. When the heavy atoms are far apart, $\left|y-x_{0}\right| \ll\left|x_{0}\right|$, Eq. (5) behaves as though there is a single $\delta$ function of modified strength at the origin. The solutions to Eq. (14) when $x_{0} \rightarrow \infty$ underestimate the correct threshold energy by $\beta /(2+\beta)$ :

$$
\lim _{|x| \rightarrow \infty} u(x)=-1-\frac{\beta}{2+\beta} .
$$

This result is not unexpected, since we have so far neglected the positive-definite contribution $\tilde{Q}(x) /\left(2 \mu_{3}\right)$ to the heavyparticle kinetic energy. It is known that neglecting this "diagonal correction"- which we call the "extreme adiabatic approximation" (EAA)-yields a lower bound $E_{\mathrm{EAA}}$ to the $N$-body bound-state energy. Including the diagonal correction, but neglecting any couplings between Born-Oppenheimer curves - an approximation we call the "uncoupled adiabatic approximation" (UAA)-yields an upper bound $E_{\mathrm{UAA}}$ to the correct energy [35-38]. We find for this problem that the trend in these inequalities $E_{\mathrm{EAA}}<E<E_{\mathrm{UAA}}$ is already present in the threshold values of the adiabatic potential itself. In other words, we find that in the limit $|x| \rightarrow \infty, u(x)<-1<$ $u(x)+\tilde{Q}(x) /\left(2 \mu_{3}\right)$. In the next section, we explicitly calculate $\tilde{Q}(x)$.

\section{B. Diagonal correction $\tilde{Q} /\left(2 \mu_{3}\right)$}

Using the solutions Eq. (13) (with $A=0$ ) along with the normalization Eq. (16), we explicitly calculate the integral involved in the nonadiabatic correction Eq. (12). Taking $A=0$ in Eq. (13), continuity of the wave function immediately yields $D=B \cosh \left(\kappa x_{0}\right) / e^{-\kappa x_{0}}$. The remaining normalization constant, $B$, depends on the $\mathrm{H}-\mathrm{H}$ separation distance both explicitly and implicitly through the eigenvalue $\kappa$ :

$$
B(x)=\frac{2 \sqrt{\kappa}}{\sqrt{2 x_{0} \kappa+e^{2 x_{0} \kappa}+1}} .
$$

Derivatives of the eigenvalue $\kappa(x)$ are replaced by expressions involving $\kappa$ itself by differentiating Eq. (14) with respect to $x$ and solving for $\kappa^{\prime}$. We find that the nonadiabatic correction $\tilde{Q}(x)$ can be expressed as a rational polynomial in the 
separation distance $x$ :

$$
\begin{aligned}
\tilde{Q}(x)= & \frac{\beta}{3(2+\beta)\left(-2 h x_{0}+2 \kappa x_{0}+1\right)^{4}}\left[3 h^{2}+x_{0}\left(-12 h^{3}\right.\right. \\
& \left.+24 h^{2} \kappa-36 h \kappa^{2}+24 \kappa^{3}\right)+x_{0}^{3}\left(-16 h^{3} \kappa^{2}\right. \\
& \left.+48 h^{2} \kappa^{3}-48 h \kappa^{4}+16 \kappa^{5}\right)+x_{0}^{2}\left(12 h^{4}-48 h^{3} \kappa\right. \\
& \left.+108 h^{2} \kappa^{2}-120 h \kappa^{3}+48 \kappa^{4}\right)+x_{0}^{4}\left(-16 h^{4} \kappa^{2}\right. \\
& \left.\left.+64 h^{3} \kappa^{3}-96 h^{2} \kappa^{4}+64 h \kappa^{5}-16 \kappa^{6}\right)\right],
\end{aligned}
$$

where we have defined the constant $h=\mu_{3} g_{3}$. Evaluating Eq. (17) at the asymptotic value of the potential Eq. (15) gives $\tilde{Q} / 2 \mu_{3} \rightarrow[\beta /(2+\beta)]+[\beta /(2+\beta)]^{2}$, and including $\tilde{Q} /\left(2 \mu_{3}\right)$ in Eq. (11) yields the correct threshold energy to order $\left(\frac{\beta}{2+\beta}\right)^{2}$ :

$$
\lim _{|x| \rightarrow \infty}\left[u(x)+\frac{\tilde{Q}}{2 \mu_{3}}\right]=-1+\left(\frac{\beta}{2+\beta}\right)^{2} .
$$

In other words, for small $\beta$, the error in the threshold energy vanishes linearly without the diagonal correction, but quadratically when it is included. Interestingly, for the equal mass case $(\beta=1)$, the UAA gives the correct threshold energy to within $11 \%$. This may seem a somewhat surprising result since the Born-Oppenheimer factorization is typically expected to fail catastrophically in this limit; however, other authors [39] have found the Born-Oppenheimer approach to work surprisingly well for short-range $s$-wave interactions in $3 \mathrm{D}$ for a wide variety of mass ratios. It seems that the present 1D calculation shares similar good fortune.

\section{Numerical results for the HHL system}

Here, we compare the present Born-Oppenheimer calculation for the HHL system to the high-accuracy calculations of [23]. Binding energies and scattering solutions are calculated in the UAA.

For the scattering calculation, $u(x)$ and $\tilde{Q}(x)$ are calculated to 15 digits on a uniform grid, and the Numerov method is used to propagate the solution out from $x=0$ to some $x_{\max }$. The attractive well in $u(x)$ widens as the mass ratio $\beta^{-1}$ increases. An $x_{\max } \sim 40$ is sufficient for $\beta^{-1} \lesssim 10$, but must be increased to $x_{\max } \sim 120$ for $\beta^{-1} \sim 250$. For a Numerov step size $s$, each integration step in the Numerov method can introduce an error of order $s^{5}$. For $N_{s}$ total steps, an upper bound to the asymptotic values of the wave function of order $N_{s} s^{5}=x_{\max } s^{4}$ is maintained less than $10^{-10}$. The asymptotic wave function is matched to

$$
\psi(x) \rightarrow \begin{cases}\cos (k x)-\tan (\delta) \sin (k x) & \text { bosons, } \\ \sin (k x)+\tan (\delta) \cos (k x) & \text { fermions. }\end{cases}
$$

The atom-dimer scattering length is then extracted from the effective range expansion as

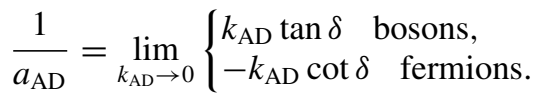

Here, $k_{\mathrm{AD}}=\sqrt{2 \mu_{23,1} E_{\text {rel }}}$, while $k=\sqrt{2 \mu_{3} E_{\text {rel }}}$. The mass ratios in Table I (discussed below) are obtained by a bisection root-finding algorithm (either on $1 / a_{\mathrm{AD}}$ or on $a_{\mathrm{AD}}$ ) to six-digit

\begin{tabular}{|c|c|c|c|c|}
\hline \multirow[b]{2}{*}{$n$} & \multicolumn{2}{|c|}{$\begin{array}{c}\lambda=0 \\
\left.\beta^{-1}\right|_{a_{\mathrm{AD}} \rightarrow 0}\end{array}$} & \multicolumn{2}{|c|}{$\begin{array}{c}\lambda=0 \\
\left.\beta^{-1}\right|_{a_{\mathrm{AD}} \rightarrow \infty}\end{array}$} \\
\hline & This work & Ref. [23] & This work & Ref. [23] \\
\hline \multicolumn{5}{|l|}{1} \\
\hline 2 & 1.357 & 0.971 & 3.255 & 2.86954 \\
\hline 3 & 9.747 & 9.365 & 12.336 & 11.9510 \\
\hline 4 & 23.333 & 22.951 & 26.602 & 26.218 \\
\hline 5 & 42.142 & 41.762 & 46.055 & 45.673 \\
\hline 6 & 66.168 & 65.791 & 70.695 & 70.317 \\
\hline 7 & 95.404 & 95.032 & 100.523 & 100.151 \\
\hline 8 & 129.845 & 129.477 & 135.539 & 135.170 \\
\hline 9 & 169.488 & 169.120 & 175.742 & 175.374 \\
\hline \multirow[t]{2}{*}{10} & 214.331 & 213.964 & 221.133 & 220.765 \\
\hline & \multicolumn{2}{|c|}{$\begin{array}{c}\lambda=\infty \\
\left.\beta^{-1}\right|_{a_{\mathrm{AD}} \rightarrow 0}\end{array}$} & \multicolumn{2}{|c|}{$\begin{array}{c}\lambda=\infty \\
\left.\beta^{-1}\right|_{a_{\mathrm{AD}} \rightarrow \infty}\end{array}$} \\
\hline$n$ & This work & Ref. [23] & This work & Ref. [23] \\
\hline 1 & & $0^{*}$ & 1.170 & $1^{*}$ \\
\hline 2 & 5.499 & 5.2107 & 7.694 & 7.3791 \\
\hline 3 & 16.456 & 16.1197 & 19.373 & 19.0289 \\
\hline 4 & 32.650 & 32.298 & 36.235 & 35.879 \\
\hline 5 & 54.067 & 53.709 & 58.283 & 57.923 \\
\hline 6 & 80.697 & 80.339 & 85.518 & 85.159 \\
\hline 7 & 112.535 & 112.179 & 117.940 & 117.583 \\
\hline 8 & 149.577 & 149.222 & 155.550 & 155.193 \\
\hline 9 & 191.820 & 191.463 & 198.347 & 197.989 \\
\hline 10 & 239.262 & 238.904 & 246.331 & 245.973 \\
\hline
\end{tabular}
precision. The number of digits reported here represents the
TABLE I. Values of the mass ratio $\beta^{-1}=m_{H} / m_{L}$ for which the atom-dimer scattering length is infinite $\left(a_{\mathrm{AD}} \rightarrow \infty\right.$, corresponding to the appearance of the $n$th trimer state), or zero $\left(a_{\mathrm{AD}} \rightarrow 0\right)$, are tabulated both in the case of noninteracting bosonic $\mathrm{H}$ atoms $(\lambda \rightarrow 0)$ and fermionic $\mathrm{H}$ atoms $(\lambda \rightarrow \infty)$. Results are compared to Ref. [23]. An asterisk $(*)$ denotes an exact result.

precision of our calculation. The accuracy is best estimated by comparing to the calculations of [23].

Bound-state calculations are performed variationally by expanding $\psi(x)$ in a basis of $b$-splines, and solving the resulting generalized eigenvalue problem. We have verified that the results are well converged with respect to the number of grid points used to interpolate the potential $u(x)$, as well as the number and placement of $b$-splines.

In Fig. 2(a), we show the three-body spectrum as a function of $\beta^{-1 / 2}$ (recall $\beta=m_{L} / m_{H}$ ). The mass ratios at which a new state appears, marked by the red crosses for $\lambda=0$ and red dots for $\lambda=\infty$, trace out a curve governed by the $\beta$ dependence of the threshold Eq. (18). In the hyperspherical calculation of [23], the threshold is reproduced exactly, and all dots and crosses appear at $E_{2} / B_{2}=-1$.

Figure 2(b) shows $\tan ^{-1}\left(a_{\mathrm{AD}} / a\right) / \pi$ as a function of $\beta^{-1 / 2}$. Again, the red dots and crosses denote the mass ratios at which a new state appears and the atom-dimer scattering length $a_{\mathrm{AD}} \rightarrow \infty$. The blue stars indicate $a_{\mathrm{AD}} \rightarrow 0$. In a manner similar to [23], we tabulate these particular values of the mass ratio in Table I. Note that the Born-Oppenheimer calculation consistently overestimates the critical mass ratios $\beta^{-1}$ by approximately $0.3-0.4$. The overestimate is understood, at least qualitatively, by noting that the UAA produces an upper 


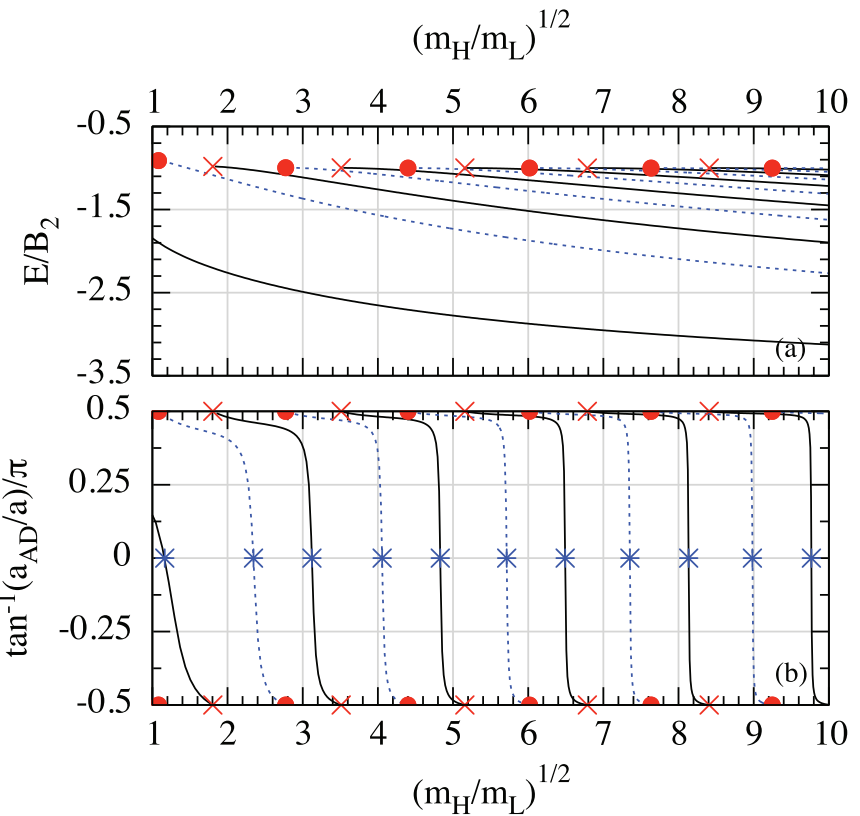

FIG. 2. (Color online) Graph (a) shows the energy spectrum for the three-body (HHL) system as a function $\beta^{-1 / 2}$. Graph (b) shows $\tan ^{-1}\left(a_{\mathrm{AD}} / a\right) / \pi$, where $a_{\mathrm{AD}}$ is the atom-dimer scattering length. For both graphs, the solid-black curves denote noninteracting bosonic $\mathrm{H}$ particles. The dashed-blue curves denote noninteracting fermionic particles, or equivalently, fermionized $\mathrm{H}$ particles with $\lambda \rightarrow \infty$. Red crosses and dots, for bosons and fermions, respectively, indicate the appearance of a new bound state and $\left|a_{\mathrm{AD}}\right| \rightarrow \infty$. Blue stars indicate $a_{\mathrm{AD}} \rightarrow 0$.

bound to the binding energy, and the trend in the spectrum is for deeper binding as $\beta^{-1}$ increases. The percentage error in the critical values of $\beta^{-1}$ decreases monotonically, as one might expect.

The $\beta=1 \mathrm{HHL}$ ground state for $\lambda=0$ bosons was found in Ref. [23] to be (in units of $B_{2}$ ) $E_{3}=-2.087719$, very close to the value $E_{3}=-2.08754$ found much earlier in Ref. [40]. Here, we find that the EAA produces a lower bound of $E_{3, \mathrm{EAA}}=-2.4227$, approximately $16 \%$ deeper than the correct value. The UAA underbinds by about $11 \%$, giving the upper bound $E_{3, \mathrm{UAA}}=-1.8561$. It is interesting that the error in the UAA calculation is almost entirely accounted for by the overestimate of the atom-dimer threshold energy. In fact, scaling by the threshold energy Eq. (18), one obtains $E_{3, \mathrm{UAA}} / E_{\text {thresh }}=-2.0879$, overbinding by only $0.01 \%$.

Kartavtsev and Malykh [41] found that universal (nonEfimov) fermionic states in $3 \mathrm{D}$ exist for mass ratios $\beta^{-1} \gtrsim$ 8.17. Pricoupenko and Pedri [42] found similar states in 2D for $\beta^{-1} \gtrsim 3.33$. Levinsen and Parish [43] established that these states are continuously connected as confinement is increased. It is interesting to speculate whether the fermionic state that appears in $1 \mathrm{D}$ at $\beta^{-1}=1\left(\beta^{-1} \approx 1.170\right.$ in our calculation $)$ is continuously connected to these universal trimer states in higher dimensions.

\section{FOUR-BODY (HHHL) PROBLEM}

Let us now turn to the calculation of four-body observables. The basic three-step recipe for this calculation is as follows.
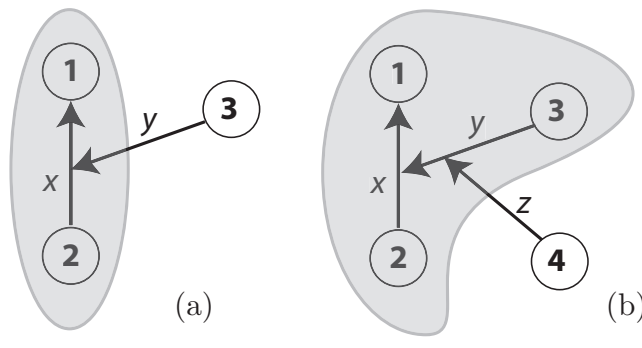

FIG. 3. Schematic diagram of the Jacobi coordinates for (a) the three-body problem and (b) the four-body problem are shown. The heavy particles are contained in the shaded regions.

First, the Born-Oppenheimer method is used to calculate the 2D potential-energy surface for the heavy particles in the extreme adiabatic approximation (EAA). Next, this potentialenergy surface is inserted into a calculation of the hyperradial adiabatic potential curves and couplings. Finally, the resulting set of coupled hyperradial equations is solved for the boundstates and atom-trimer scattering length. The entire procedure is then repeated for different values of $\beta$. If a sufficiently large number of hyperradial curves and couplings are included in the final step, then the accuracy of the calculation is limited almost entirely by the EAA made in the first step.

\section{A. Adiabatic equations}

For all four-body (HHHL) calculations that follow, we choose particles 1,2 , and 3 to have mass $m_{1}=m_{2}=m_{3}=$ $m_{H}$ and particle 4 to have mass $m_{4}=\beta m_{H}$. The solution to the adiabatic equation is most easily carried out using the "K-type" Jacobi coordinates shown in Fig. 3(b), with unitless mass-scaled coordinates defined as

$$
\begin{aligned}
& x=\frac{1}{a} \sqrt{\frac{\mu_{12}}{\mu_{4 b}}}\left(x_{1}-x_{2}\right), \\
& y=\frac{1}{a} \sqrt{\frac{\mu_{12,3}}{\mu_{4 b}}}\left(\frac{m_{1} x_{1}+m_{2} x_{2}}{m_{1}+m_{2}}-x_{3}\right), \\
& z=\frac{1}{a} \sqrt{\frac{\mu_{123,4}}{\mu_{4 b}}}\left(\frac{m_{1} x_{1}+m_{2} x_{2}+m_{3} x_{3}}{m_{1}+m_{2}+m_{3}}-x_{4}\right) .
\end{aligned}
$$

Here, $\mu_{4 b}=\left(\mu_{12} \mu_{12,3} \mu_{123,4}\right)^{1 / 3}$ is the four-body reduced mass. Again, we rescale the Schrödinger equation by the heavy-light binding energy $B_{2}$. The full four-body Schrödinger equation then reads

$$
\begin{aligned}
& \frac{-1}{2 \mu_{4}} \nabla^{2} \Psi(\rho, \phi, z)+\left[g_{4} \sum_{i=1}^{3} \delta\left(z-z_{i}\right)\right. \\
& \left.\quad+\lambda g_{4} \sum_{i<j}^{3} \delta\left(\alpha \rho\left|\sin \left(\phi-\phi_{i j}\right)\right|\right)\right] \Psi(\rho, \phi, z) \\
& =E \Psi(\rho, \phi, z),
\end{aligned}
$$

where $\alpha=\sqrt{6}[(3+\beta) / \beta]^{1 / 6}, \phi_{12}=\pi / 2$, and $\phi_{23}=-\phi_{13}=$ $\pi / 6$. Again, rescaling by $B_{2}$ introduces the following unitless 
parameters:

$$
\begin{aligned}
\mu_{4} & =\frac{\beta+1}{2 \beta^{2 / 3}(3+\beta)^{1 / 3}}, \\
g_{4} & =-2 \sqrt{3}\left(\frac{\beta}{3+\beta}\right)^{1 / 3}, \\
z_{i} & =\sqrt{\frac{2 \beta}{3+\beta} \rho \sin \left(\phi-\phi_{i}\right),}
\end{aligned}
$$

where $\phi_{1}=-4 \pi / 3, \phi_{2}=0$, and $\phi_{3}=-2 \pi / 3$. The particular choice of Jacobi coordinates Eq. (21) has the advantage that the separation distances $x_{12}, x_{13}$, and $x_{23}$ are all independent of the $z$ coordinate. The heavy-particle dynamics is restricted to the $x-y$ plane, and the light particle can be integrated out by solving an equation in the $z$ coordinate only, with fixed $x$ and $y$. The transformation to hyperspherical coordinates is accomplished by expressing $x, y$, and $z$ in terms of the usual spherical polar coordinates $R, \theta$, and $\phi$. The heavy-particle subsector is then described by $x=\rho \cos \phi$ and $y=\rho \sin \phi$, where $\rho=\sqrt{x^{2}+y^{2}}$ is the projection of $R$ onto the $x$-y plane: $\rho=R \sin \theta$ and $z=R \cos \theta$.

Clearly, fixing $x$ and $y$ is equivalent to fixing $\rho$ and $\phi$. We make the Born-Oppenheimer factorization:

$$
\Psi=\Phi(\rho, \phi ; z) \psi(\rho, \phi),
$$

where the adiabatic equation for the Born-Oppenheimer surface is

$$
\left(\frac{-1}{2 \mu_{4}} \frac{\partial^{2}}{\partial z^{2}}+g_{4} \sum_{i=1}^{3} \delta\left(z-z_{i}\right)\right) \Phi(\rho, \phi ; z)=U(\rho, \phi) \Phi(\rho, \phi ; z) .
$$

The heavy-particle eigenstates now live on the potentialenergy surface $U(\rho, \phi)$, and satisfy (in the EAA)

$$
\begin{aligned}
& \frac{-1}{2 \mu_{4}}\left(\frac{1}{\rho} \frac{\partial}{\partial \rho} \rho \frac{\partial}{\partial \rho}+\frac{1}{\rho^{2}} \frac{\partial^{2}}{\partial \phi^{2}}\right) \psi(\rho, \phi) \\
& \quad+\left[U(\rho, \phi)+\lambda g_{4} \sum_{i<j}^{3} \delta\left(\alpha \rho\left|\sin \left(\phi-\phi_{i j}\right)\right|\right)\right] \psi(\rho, \phi) \\
& =E_{\text {EAA }} \psi(\rho, \phi)
\end{aligned}
$$

Finally, we describe $\psi(\rho, \phi)$ as a sum over adiabatic channel functions:

$$
\psi(\rho, \phi)=\sum_{n=0}^{\infty} \rho^{-1 / 2} f_{n}(\rho) \chi_{n}(\rho ; \phi)
$$

where $\chi_{n}(\rho ; \phi)$ satisfy the fixed- $\rho$ equation:

$$
\begin{aligned}
& \frac{-1}{2 \mu_{4} \rho^{2}} \frac{\partial^{2} \chi_{n}}{\partial \phi^{2}}+U(\rho, \phi) \chi_{n}+\lambda g_{4} \sum_{i<j}^{3} \delta\left(\alpha \rho\left|\sin \left(\phi-\phi_{i j}\right)\right|\right) \chi_{n} \\
& =U_{n}(\rho) \chi_{n} .
\end{aligned}
$$

Because we only consider $\lambda=0$ and $\lambda \rightarrow \infty$, the $\delta$ functions in Eq. (30) result in simple boundary conditions at $\phi=\pi / 6$. For arbitrary $\lambda$, one would need to account for the $\lambda$-dependent derivative discontinuity at $\phi=\pi / 6$. Inserting the expansion Eq. (29) into Eq. (28) results in a set of coupled equations in $\rho$, which are conveniently written in matrix form as

$$
\begin{aligned}
& \frac{-1}{2 \mu_{4}}\left(\mathbf{1} \frac{\partial^{2}}{\partial \rho^{2}}+\mathbf{Q}(\rho)+2 \mathbf{P}(\rho) \frac{\partial}{\partial \rho}\right) \vec{f}(\rho)+\mathbf{U}_{\text {eff }}(\rho) \vec{f}(\rho) \\
& =E_{\text {EAA }} \vec{f}(\rho) .
\end{aligned}
$$

Here, $\mathbf{U}_{\text {eff }}$ is a diagonal matrix with elements $U_{n}(\rho)-$ $1 / 8 \mu_{4} \rho^{2}, \quad P_{m n}(\rho)=\left\langle\chi_{m} \mid \frac{\partial \chi_{n}}{\partial \rho}\right\rangle_{\phi}$, and $Q_{m n}(\rho)=\left\langle\chi_{m} \mid \frac{\partial^{2} \chi_{n}}{\partial \rho^{2}}\right\rangle_{\phi}$. When $\mathbf{P}$ and $\mathbf{Q}$ are included in the solution to Eq. (31), and enough channels are retained for numerical convergence, the accuracy of the four-body energy is (in principle) limited only by the omission of first and second derivative couplings, $\left\langle\Phi_{m} \mid \vec{\nabla} \Phi_{n}\right\rangle_{z}$ and $\left\langle\Phi_{m} \mid \nabla^{2} \Phi_{n}\right\rangle_{z}$, that arise from generalizing Eq. (26) to include a sum: $\Psi=\sum_{n} \Phi_{n} \psi_{n}$. Such a generalization is not possible for our model without the introduction of a confining potential because Eq. (27) admits only one solution that vanishes as $|z| \rightarrow \infty$.

Identical particle symmetry of the heavy particles allows one to restrict the domain of the four-body wave function to the region $0<\phi<\pi / 6$. Thus, for a given permutation of heavy particles, the locus of points describing the coalescence of a heavy particle and a light particle-i.e., when $z$ is equal to $z_{i}$-remain ordered $z_{1}<z_{2}<z_{3}$ along the $z$ coordinate. Because the ordering is independent of $\rho$ and $\phi$, the solution to Eq. (27) for all $\rho$ and all $\phi \in[0, \pi / 6]$ is straightforward.

The boundary condition on $\chi(\rho ; \phi)$ at $\phi=0$ is determined by a combination of the parity operator, $\hat{\Pi} \phi \rightarrow \phi+\pi$, and the 1-2 permutation operator, $\hat{P}_{12} \phi \rightarrow \pi-\phi$, by the rule: $\hat{P}_{12} \hat{\Pi} \phi \rightarrow-\phi$. Considering positive parity, the boundary conditions on $\chi(\rho ; \phi)$ for noninteracting bosons are $\left.\frac{\partial \chi}{\partial \phi}\right|_{\phi=0}=\left.\frac{\partial \chi}{\partial \phi}\right|_{\phi=\pi / 6}=0$, while for noninteracting fermions, $\left.\chi\right|_{\phi=0}=\left.\chi\right|_{\phi=\pi / 6}=0$. For negative parity noninteracting bosons, we impose $\left.\chi\right|_{\phi=0}=\left.\frac{\partial \chi}{\partial \phi}\right|_{\phi=\pi / 6}=0$, while for negative parity noninteracting fermions, $\left.\frac{\partial \chi}{\partial \phi}\right|_{\phi=0}=\left.\chi\right|_{\phi=\pi / 6}=0$. Note that the boundary conditions for noninteracting fermions of positive parity are equivalent to those for bosons of negative parity, but $\lambda \rightarrow \infty$.

\section{B. Numerical solutions for the HHHL system}

In the Appendix, we calculate the transcendental equation for the eigenvalue of a 1D Schrödinger equation with three $\delta$ functions of arbitrary strength and arbitrary placement. The resulting Eq. (A3) is applied to the Eq. (27) by letting $g_{a}=g_{b}=g_{c}=2 \mu_{4} g_{4}, \kappa^{2}=-2 \mu_{4} U(\rho, \phi)>0$, and $a=z_{1}$, $b=z_{2}$, and $c=z_{3}$.

Figure 4 shows the potential-energy surface for the particular mass ratio $\beta^{-1}=22.08$ appropriate for an atomic mixture of Li-Cs. Potential surfaces like this one are calculated by solving Eq. (A3) on a nonlinear grid with typically $200 \times 400$ points in the $(\rho, \phi)$ plane. The points are distributed so that more grid points are concentrated in the vicinity of the well at $\rho=0$ and the valley near $\phi=\pi / 6$. Particular care must be taken to describe the valley near $\phi=\pi / 6$ accurately at large $\rho$, or else the numerical solution to the fixed- $\rho$ adiabatic equation does not reproduce the correct threshold behavior in any of the atom-trimer channels. This is because the fixed- $\rho$ solutions as $\rho \rightarrow \infty$ should approach the HHL bound-state energies from the spectrum in Fig. 2 with the correct $\rho$ dependence. In 


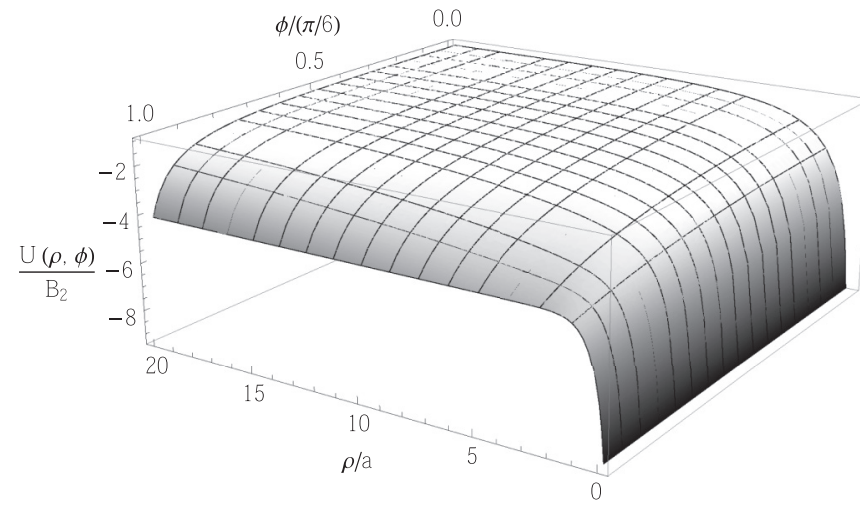

FIG. 4. Born-Oppenheimer surface for the HHHL system is shown. $U(\rho, \phi)$ is in units of $B_{2}$ and $\rho$ is in units of $a$.

particular, at large $\rho$ we find that $Q_{00}(\rho) / 2 \mu_{4} \rightarrow-1 / 8 \mu_{4} \rho^{2}$,

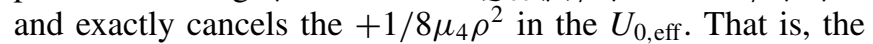
effective potential with the diagonal correction approaches a constant, and describes a two-body channel to which Eqs. (19) and (20) may be applied with the replacements $\psi \rightarrow f$, $k_{\mathrm{AD}} \rightarrow k_{\mathrm{AT}}=\sqrt{2 \mu_{234,1} E_{\mathrm{rel}}}, a_{\mathrm{AD}} \rightarrow a_{\mathrm{AT}}$, and $x \rightarrow \rho$, along with the boundary condition $f(0)=0$.

Figures 1(a) and 1(c) show the hyperradial potential curves $U_{n}(\rho)$ obtained by solving the fixed- $\rho$ Eq. (30) using the potential-energy surface shown in Fig. 4. Note that, at large $\rho$, the lowest potential curves converge to the appropriate HHL bound-state energy shown as red dashed lines in Figs. 1(b) and $1(\mathrm{~d})$, as appropriate for an atom-trimer channel. The red dashed lines in Figs. 1(a) and 1(c) indicate HHHL bound states obtained by solving Eq. (31) with 10 coupled channels. Typically, calculations with only the lowest channel (but including the diagonal correction) give bound-state energies converged to four or five digits. The error incurred by ignoring excited hyperradial potential curves is expected to be negligible compared to making the EAA in the calculation of the surface $U(\rho, \phi)$.

In Figs. 5 and 6, we show the spectrum and atom-trimer scattering lengths of the HHHL system with noninteracting bosonic and fermionic heavy atoms, respectively. We show both positive $(\mathrm{B}+, \mathrm{F}+)$, and negative $(\mathrm{B}-, \mathrm{F}-)$ parity cases for each identical particle symmetry. The HHL ground-state energies for each symmetry from Fig. 2(a) are replotted here as dashed-red curves. Again, mass ratios at which a new tetramer state appears (and $\left|a_{\mathrm{AT}}\right| \rightarrow \infty$ ) are marked by red crosses, while zeros of $a_{\mathrm{AT}}$ are indicated by blue stars. The particular numerical values for the coordinates $\left(\beta^{-1 / 2}, E / B_{2}\right)$ are also marked. As the mass-ratio $\beta^{-1}$ increases, four-body bound states enter at lower energies than one would expect from the three-body calculation (i.e., the dashed-red curve). This discrepancy in the threshold energy is attributed to the fact that the EAA underestimates the potential surface in Fig. 4 by neglecting the positive nonadiabatic correction $-\left\langle\Phi \mid \nabla^{2} \Phi\right\rangle_{z} / 2 \mu_{4}$, while the corresponding correction is included at the three-body level in Fig. 2.

While we perform a multichannel calculation of the spectrum, we find that it is sufficient to use a simple single-channel calculation for $a_{\mathrm{AT}}$. Indeed, comparing the critical mass ratios for which $a_{\mathrm{AT}} \rightarrow \infty$, and a tetramer state lies at threshold, we

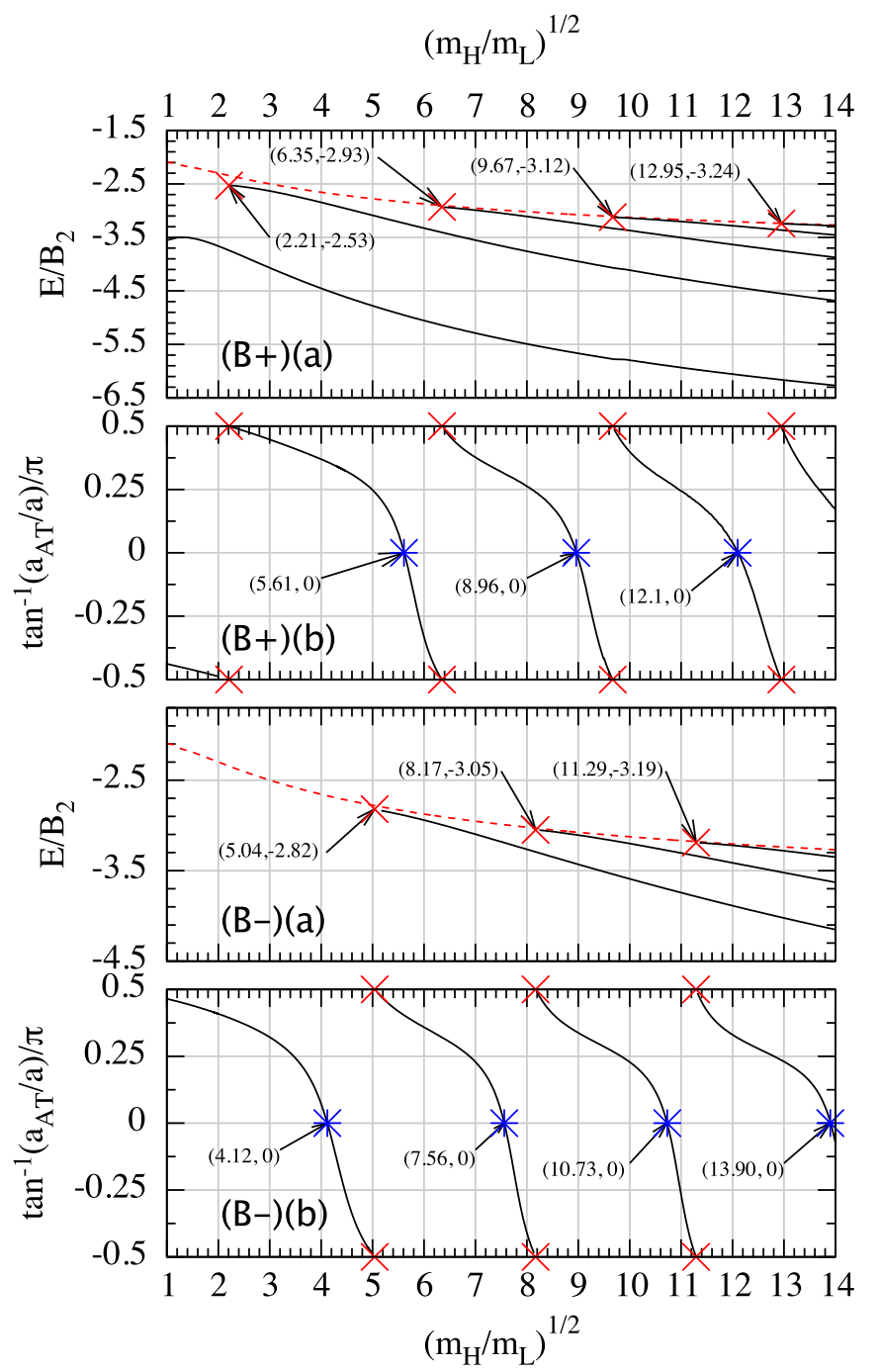

FIG. 5. (Color online) Graphs $(B+)(a)$ and $(B-)(a)$ show the HHHL spectrum for bosonic heavy particles of positive and negative parity, respectively. The red crosses indicate the appearance of a new HHHL bound state and $a_{\mathrm{AT}} \rightarrow \infty$. The red dashed curve is the lowest (solid line) bosonic HHL bound state from Fig. 2(a). Graphs $(\mathrm{B}+)(\mathrm{b})$ and $(\mathrm{B}-)(\mathrm{b})$ show the arctangent of the atom-trimer scattering length for bosonic heavy particles of positive and negative parity, respectively. The blue stars indicate $a_{\mathrm{AT}} \rightarrow 0$. The specific ordinates of the crosses and stars are labeled.

find good agreement between the two calculations. This can be readily observed by comparing the positions of the red crosses in graphs (a) and (b) of Fig. 5, and similarly in Fig. 6.

At $\beta=1$, we find that noninteracting bosons of positive parity admit an HHHL bound state with $E_{4 \text {,EAA }} \approx-3.55$. The second tetramer state appears at $\beta^{-1} \approx 4.88$, and the third at $\beta^{-1} \approx 40.3$. For Li-Cs mixtures, one might expect two universal tetramer states. Negative-parity bosons are less likely to bind than those with positive parity. The first tetramer state appears at $\beta^{-1} \approx 25.4$, and the second at $\beta^{-1} \approx 66.7$.

For fermionic particles, negative-parity tetramers are more likely to bind than those with positive parity. The precise value of the critical mass ratio is difficult to pin down within the Born-Oppenheimer approximation at these small mass 


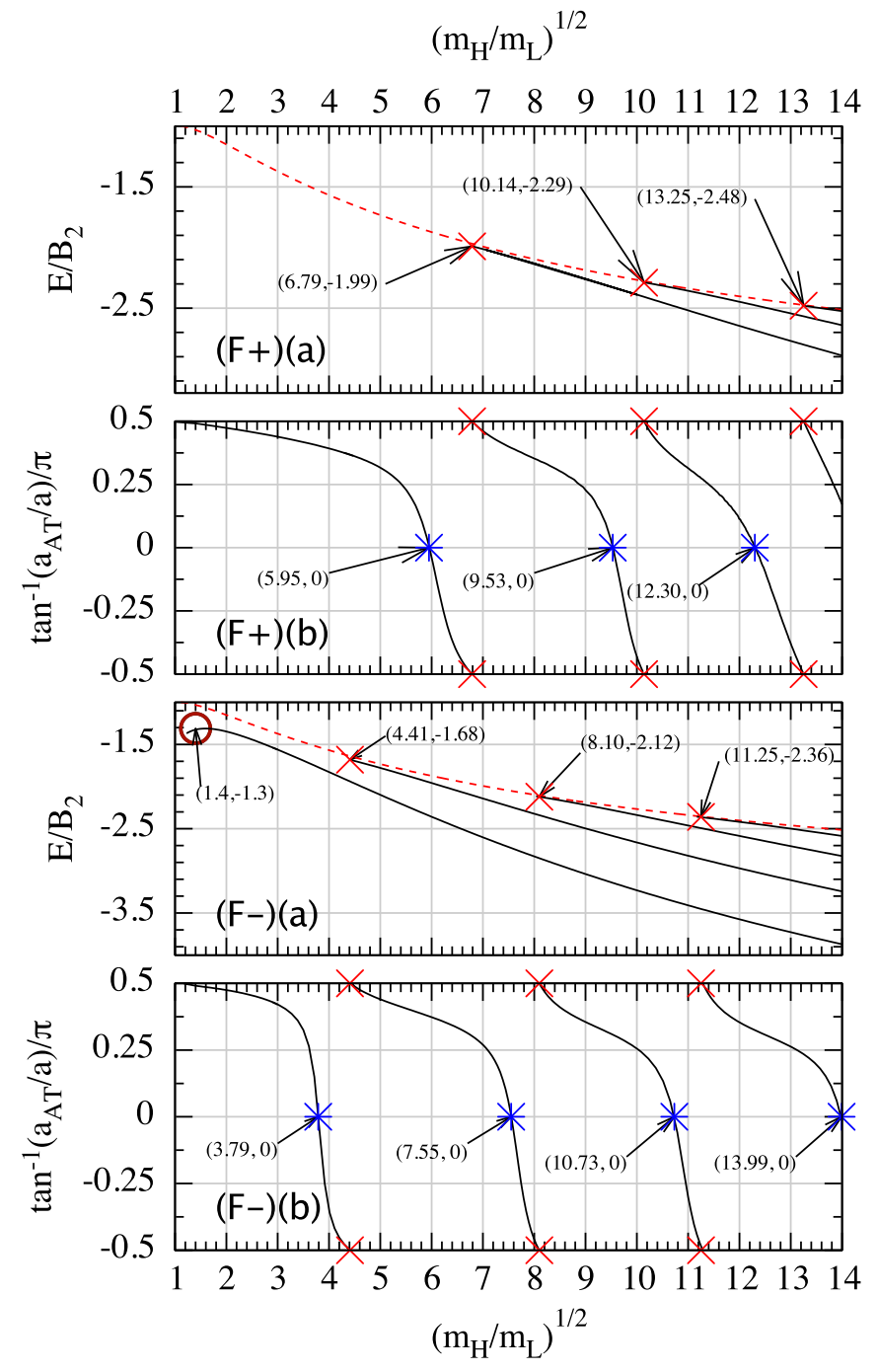

FIG. 6. (Color online) Graphs $(\mathrm{F}+)(\mathrm{a})$ and $(\mathrm{F}-)(\mathrm{a})$ show the HHHL spectrum for fermionic heavy particles of positive and negative parity, respectively. The red crosses indicate the appearance of a new HHHL bound state and $a_{\mathrm{AT}} \rightarrow \infty$. The red dashed curve is the lowest (solid line) fermionic HHL bound state from Fig. 2(a). Graphs $(\mathrm{F}+)(\mathrm{b})$ and $(\mathrm{F}-)(\mathrm{b})$ show the arctangent of the atom-trimer scattering length for fermionic heavy particles of positive and negative parity, respectively. The blue stars indicate $a_{\mathrm{AT}} \rightarrow 0$. The specific ordinates of the crosses and stars are labeled.

ratios. The difficulty is magnified because even in the UAA three-body calculations of Sec. II, the trimer state doesn't appear until $\beta^{-1} \approx 1.17$. Because the four-body calculation doesn't include the positive nonadiabatic correction to the potential-energy surface shown in Fig. 4, one expects the tetramer bound state to appear below the atom-trimer threshold prematurely. In the four-body calculation, the energy of the atom-trimer threshold itself increases slightly with $\beta^{-1 / 2}$, and the tetramer energy tracks along with it until about $\beta^{-1} \approx 2.6$. The increasing threshold energy is undoubtedly an artifact of the approximation at the four-body level since it is absent in the more accurate three-body calculations. We can nonetheless estimate the critical mass ratio as $\beta^{-1} \approx 2.0 \pm 0.6$, indicated by a red circle in Fig. $6(\mathrm{~F}-)(\mathrm{a})$. The second negative-parity fermionic state appears at $\beta^{-1}=19.4$.
In 3D, Blume [44] found that a universal tetramer exists for fermionic particles above a mass ratio of $\beta^{-1} \approx 9.5$. In 2D, Levinsen and Parish [43] found a critical mass ratio of $\beta^{-1} \approx 5$.0. It is interesting to speculate whether these states are continuously connected to each other, and to the universal state that appears in these calculations at $\beta^{-1} \approx 2.0$ for negativeparity fermions.

\section{SUMMARY AND OUTLOOK}

We have calculated three-body and four-body spectra, as well as the atom-dimer and atom-trimer scattering lengths for two-component systems with one light particle, as a function of the mass ratio. Heavy particles are assumed to be noninteracting, and the four-particle system is assumed to be in free space. Both bosonic and fermionic heavy particles are treated. For the HHL system, the Born-Oppenheimer method gives good quantitative agreement with the hyperspherical calculations of [23]. For the HHHL system, the potential-energy surface governing the heavy-particle dynamics is calculated in the "extreme adiabatic approximation." That surface is then used to calculate hyperradial potential curves and couplings. The values for the resulting atom-trimer thresholds converge to the appropriate three-body bound-state energies, lending some confidence to the HHHL calculation.

Let us now discuss possible extensions of this work. Note that we have scaled away the only length scale, $a$, that appears in our model. There are two immediate generalizations that expand the parameter space considerably.

First, there is the generalization to arbitrary H-H interactions, which introduces the H-H scattering length $a_{H H}$. Such an extension was already treated at the three-body level in Ref. [23], but no such four-body calculations have appeared in the literature. In a hyperspherical calculation, the additional derivative discontinuity in the angular wave function is treated analytically, and the hyperradial potential curves are calculated as the solution to a single transcendental equation $[21,23]$. The HHHL Born-Oppenheimer calculation for bosons can be extended to arbitrary $\lambda$ by choosing a $b$-spline basis set that satisfies the boundary condition,

$$
\left.\lim _{\epsilon \rightarrow 0} \frac{1}{\chi} \frac{\partial \chi}{\partial \phi}\right|_{\pi / 6-\epsilon}=\frac{\rho \lambda(1+\beta)}{\sqrt{2} \beta}\left(\frac{\beta}{3+\beta}\right)^{5 / 6} .
$$

With this generalization, one can smoothly transition between the energies shown in Figs. 5 and 6, passing from noninteracting bosons to the fermionized limit.

The bound-state calculation can be extended by the introduction of a harmonic trapping potential, which separates into relative and center-of-mass parts under the transformation to Jacobi coordinates. This extension would establish a connection with several papers that have appeared recently, treating equal-mass two-component systems [45-47]. The addition of a trapping potential would introduce excited potential-energy surfaces and the possibility of interesting physics beyond the Born-Oppenheimer approximation.

Here, we have only considered the " $3+1$ " branch (i.e., the HHHL system) of the few-component problem. It may be that other branches can be treated by similar methods. For example, for the 2+2 (HHLL) problem, integrating out the light atoms would result in a $1 \mathrm{D}$ heavy-heavy potential, but the adiabatic 
equation is a 2D partial differential equation, instead of a 1D equation like Eq. (27).

Finally, it is worth emphasizing that ultimately a fully $3 \mathrm{D}$ solution to the few-body problem with finite-range interactions is needed in order to understand the physics of quasi-1D systems. A hyperspherical solution to the few-body problem in quasi-1D for finite-range interactions remains a significant challenge, although recent advances in the correlated Gaussian hyperspherical method [48] may make these calculations possible.

\section{ACKNOWLEDGMENTS}

I would like to thank Brett Esry, Chris Greene, and Jose D'Incao for early discussions related to this topic. Thanks also to Jesper Levinsen for a series of helpful correspondences.

\section{APPENDIX: TRIPLE $\delta$-FUNCTION PROBLEM}

Here, we give the solution for the eigenvalue to the following Schrödinger equation:

$$
\begin{aligned}
& {\left[-\frac{\partial^{2}}{\partial z^{2}}+g_{a} \delta(z-a)+g_{b} \delta(z-b)+g_{c} \delta(z-c)\right] \Phi(z)} \\
& \quad=-\kappa^{2} \Phi(z) .
\end{aligned}
$$

We assume that the positions of the $\delta$ functions are ordered as $a<b<c$, but no other assumptions regarding their placement are made. In particular, the Hamiltonian is not assumed to commute with the parity operator. The (unnormalized) solution satisfying the asymptotic boundary condition, $\Phi(|z| \rightarrow \infty)=$ 0 , is elementary:

$$
\Phi(z)=\left\{\begin{aligned}
\Phi_{\mathrm{I}} & =A e^{\kappa z}, & & z<a, \\
\Phi_{\mathrm{II}} & =B e^{-\kappa z}+C e^{\kappa z}, & & a<z<b, \\
\Phi_{\mathrm{III}} & =D e^{-\kappa z}+E e^{\kappa z}, & & b<z<c, \\
\Phi_{\mathrm{IV}} & =F e^{-\kappa z}, & & c<z .
\end{aligned}\right.
$$

Matching the solutions and enforcing the derivative discontinuities at $z=a, z=b$, and $z=c$ yields, after considerable algebra,

$$
\begin{aligned}
& g_{a} g_{c}\left(g_{b}-2 \kappa\right) e^{2 \kappa(a+b)}-g_{a} g_{b}\left(g_{c}+2 \kappa\right) e^{2 \kappa(a+c)} \\
& \quad+\left(g_{a}+2 \kappa\right)\left(g_{b}+2 \kappa\right)\left(g_{c}+2 \kappa\right) e^{2 \kappa(b+c)} \\
& -g_{b} g_{c} e^{4 b \kappa}\left(g_{a}+2 \kappa\right)=0 .
\end{aligned}
$$

For the special case of a quadrupolar potential $(a=-c, b=0$, and $g_{a}=g_{c}=-g_{b} / 2$ ), Eq. (A3) reduces to the result found recently by Patil [49].
[1] M. Greiner, I. Bloch, O. Mandel, T. W. Hänsch, and T. Esslinger, Phys. Rev. Lett. 87, 160405 (2001).

[2] A. Görlitz, J. M. Vogels, A. E. Leanhardt, C. Raman, T. L. Gustavson, J. R. Abo-Shaeer, A. P. Chikkatur, S. Gupta, S. Inouye, T. Rosenband, and W. Ketterle, Phys. Rev. Lett. 87, 130402 (2001).

[3] B. Laburthe Tolra, K. M. O’Hara, J. H. Huckans, W. D. Phillips, S. L. Rolston, and J. V. Porto, Phys. Rev. Lett. 92, 190401 (2004).

[4] T. Kinoshita, T. Wenger, and D. S. Weiss, Science 305, 1125 (2004).

[5] T. Kinoshita, T. Wenger, and D. S. Weiss, Nature (London) 440, 900 (2006).

[6] A. E. Leanhardt, A. P. Chikkatur, D. Kielpinski, Y. Shin, T. L. Gustavson, W. Ketterle, and D. E. Pritchard, Phys. Rev. Lett. 89, 040401 (2002)

[7] D. G. Fried, T. C. Killian, L. Willmann, D. Landhuis, S. C. Moss, D. Kleppner, and T. J. Greytak, Phys. Rev. Lett. 81, 3811 (1998).

[8] R. H. Parker, M. R. Dietrich, K. Bailey, J. P. Greene, R. J. Holt, M. R. Kalita, W. Korsch, Z.-T. Lu, P. Mueller, T. P. O'Connor, J. Singh, I. A. Sulai, and W. L. Trimble, Phys. Rev. C 86, 065503 (2012).

[9] K.-K. Ni, S. Ospelkaus, M. De Miranda, A. Pe'er, B. Neyenhuis, J. Zirbel, S. Kotochigova, P. Julienne, D. Jin, and J. Ye, Science 322, 231 (2008).

[10] G. Barontini, C. Weber, F. Rabatti, J. Catani, G. Thalhammer, M. Inguscio, and F. Minardi, Phys. Rev. Lett. 103, 043201 (2009).

[11] H. Hara, Y. Takasu, Y. Yamaoka, J. M. Doyle, and Y. Takahashi, Phys. Rev. Lett. 106, 205304 (2011).

[12] E. Lieb and W. Liniger, Phys. Rev. 130, 1605 (1963).

[13] J. B. McGuire, J. Math. Phys. 5, 622 (1964).
[14] C. N. Yang, Phys. Rev. Lett. 19, 1312 (1967).

[15] C. Yang and C. Yang, J. Math. Phys. 10, 1115 (1969).

[16] L. Dodd, J. Math. Phys. 11, 207 (1970).

[17] H. B. Thacker, Phys. Rev. D 11, 838 (1974)

[18] W. G. Gibson, S. Y. Larsen, and J. Popiel, Phys. Rev. A 35, 4919 (1987).

[19] A. Amaya-Tapia, S. Y. Larsen, and J. Popiel, Few-Body Syst. 23, 87 (1998).

[20] N. P. Mehta and J. R. Shepard, Phys. Rev. A 72, 032728 (2005).

[21] N. P. Mehta, B. D. Esry, and C. H. Greene, Phys. Rev. A 76, 022711 (2007).

[22] O. Chuluunbaatar, A. Gusev, M. Kaschiev, V. Kaschieva, A. Amaya-Tapia, S. Larsen, and S. Vinitsky, J. Phys. B: At. Mol. Opt. Phys. 39, 243 (2006).

[23] O. I. Kartavtsev, A. V. Malykh, and S. A. Sofianos, Zh. Eksp. Teor. Fiz. 135, 419 (2009).

[24] G. Orso, E. Burovski, and T. Jolicoeur, Phys. Rev. Lett. 104, 065301 (2010).

[25] J. H. Macek, J. Phys. B: At. Mol. Opt. Phys. 1, 831 (1968).

[26] Y. Castin, C. Mora, and L. Pricoupenko, Phys. Rev. Lett. 105, 223201 (2010).

[27] N. L. Guevara, Y. Wang, and B. D. Esry, Phys. Rev. Lett. 108, 213202 (2012).

[28] D. S. Petrov, G. E. Astrakharchik, D. J. Papoular, C. Salomon, and G. V. Shlyapnikov, Phys. Rev. Lett. 99, 130407 (2007).

[29] M. Olshanii, Phys. Rev. Lett. 81, 938 (1998).

[30] T. Bergeman, M. G. Moore, and M. Olshanii, Phys. Rev. Lett. 91, 163201 (2003).

[31] C. Mora, R. Egger, and A. O. Gogolin, Phys. Rev. A 71, 052705 (2005).

[32] C. Mora, A. Komnik, R. Egger, and A. O. Gogolin, Phys. Rev. Lett. 95, 080403 (2005). 
[33] J. Levinsen, P. Massignan, and M. Parish, arXiv:1402.1859 [cond-mat.quant-gas].

[34] M. D. Girardeau, J. Math Phys. 1, 516 (1960).

[35] V. F. Brattsev, Sov. Phys.-Dokl. 10, 44 (1965).

[36] S. T. Epstein, J. Chem. Phys. 44, 836 (1966).

[37] A. F. Starace and G. L. Webster, Phys. Rev. A 19, 1629 (1979).

[38] H. T. Coelho and J. E. Hornos, Phys. Rev. A 43, 6379 (1991).

[39] S. K. Adhikari, V. Brito, H. Coelho, and T. Das, Nuovo Cimento B Ser. 11 107, 77 (1992).

[40] M. Gaudin and B. Derrida, J. Phys. 36, 1183 (1975).

[41] O. Kartavstev and A. Malykh, J. Phys. B: At. Mol. Opt. Phys. 40, 1429 (2007)
[42] L. Pricoupenko and P. Pedri, Phys. Rev. A 82, 033625 (2010).

[43] J. Levinsen and M. M. Parish, Phys. Rev. Lett. 110, 055304 (2013).

[44] D. Blume, Phys. Rev. Lett. 109, 230404 (2012).

[45] S. E. Gharashi and D. Blume, Phys. Rev. Lett. 111, 045302 (2013).

[46] T. Sowiński, T. Grass, O. Dutta, and M. Lewenstein, Phys. Rev. A 88, 033607 (2013).

[47] E. J. Lindgren, J. Rotureau, C. Forssn, A. G. Volosniev, and N. T. Zinner, arXiv:1304.2992v1 [cond-mat].

[48] K. M. Daily and C. H. Greene, Phys. Rev. A 89, 012503 (2014). [49] S. Patil, Eur. J. Phys. 30, 629 (2009). 